\title{
The Web of Radios - Introducing African Community Radio as an interface to the Web of Data
}

\author{
Anna Bon ${ }^{1}$, Victor de Boer ${ }^{1}$, Pieter De Leenheer ${ }^{1}$, Chris van Aart ${ }^{1}$, Nana Baah \\ Gyan $^{1}$, Max Froumentin ${ }^{2}$, Stephane Boyera ${ }^{2}$, Mary Allen ${ }^{3}$, Hans Akkermans ${ }^{1}$ \\ 1 Network Institute, VU University, Amsterdam, The Netherlands \\ \{a.bon, v.de.boer, pieter.de.leenheer, c.j.van.aart, \\ n.b.gyan\}@vu.nl, hans.akkermanseakmc.nl \\ 2 World Wide Web Foundation \\ $\{$ maxf, boyera\} @webfoundation. org \\ 3 Sahel Eco, ACI 200 Rue 402, 03 BP 259, Bamako, Mali \\ mary.sahelecodafribonemali.net
}

\begin{abstract}
The World Wide Web as it is currently deployed can only be accessed using modern client devices and graphical interfaces, within an infrastructure compassing datacenters and reliable, high speed Internet connections. However, in many regions in developing countries these conditions are absent. Many people living in remote rural areas in developing countries will not be able to use the Web, unless they can produce and consume voice-based content using alternative interfaces such as $(2 \mathrm{G})$ mobile phone, and radio. In this paper we introduce a radio platform, based on a use case and requirements analysis of community radio stations in Mali. The voice-based content of this radio platform will be made publicly available, using Linked Data principles, and will be ready for unexpected re-use. It will help to bring the benefits of the Web to people who are out of reach of computers and the Internet.
\end{abstract}

Keywords: community radio, voice-based interfaces, Web of Data, radio platform

\section{Introduction}

The World Wide Web is perfectly adapted for use by people in developed countries. It is visual, text-based, and mainly written in English or a few other world languages ${ }^{4}$. The Web depends on the availability of computers, datacenters, glass fiber backbones, fixed and wireless networks, $3 \mathrm{G}$ mobile telephony and transport of large volumes of data at high speed. In remote rural areas in many developing countries, conditions are different. Poor infrastructure, lack of equipment, low levels of literacy, and use of under-resourced local languages, seriously hamper the access to the Web for many people.

There is a general consensus that the global Information Society must benefit all people in the world. The United Nations Millennium Declaration contains a commitment for developing a people-centred, inclusive and development-oriented Information Society

\footnotetext{
${ }^{4}$ Wikipedia, http://en.wikipedia.org/wiki/Global_Internet_usage,Global Internet Usage
} 
so that people everywhere can create, access, utilize and share information and knowledge to attain the internationally agreed development goals and objectives, including the Millennium Development Goals. ${ }^{5}$

Yet, in many rural regions in Africa community radio is the only source of information. People have radios at home and listen to programs broadcast in local languages every day. Many people have access to simple voice-based $(2 \mathrm{G})$ mobile phone, but text messaging is hardly used [1].

The availability of both mobile phone and radio is opening opportunities for new services. E.g. radio listeners phone to the radio station and leave voice messages that they want to have broadcast, or react to popular radio programs leaving news, opinion, regional information etc. Community radio here operates as an important local information hub, where people bring information for further dissemination.

Radio stations in rural areas in Africa operate under harsh conditions. Only the largest and state financed radio stations have a computer and an internet connection. Due to lack of funds many radio stations still use old-fashioned, analogue equipment, such as tape recorders. Yet, it is in the line of expectation that more and more radio stations will have computers and an internet connection in the coming years.

In the current situation the information broadcast by the community radio is volatile: it is not stored and kept for later access or re-use. Radios do not have means to manage, reuse and index this voice-based content.

In this paper we introduce a radio platform as a new interface to the Web. It enables management of radio content in an efficient way, making it accessible and searchable, so that it can serve a broad audience, e.g. Africans in the diaspora, who want to have news from their home villages 6

Additional, the voice-based radio content on this radio platform might be linked to other data sources on the Web, enabling community radios in Africa to become an interface to the Web of Data. An example of a system that manages market information based on Linked Data principles and produces voice-output as broadcasts for African community radios, is described by De Boer et al. [2]. In the future new applications providing locally relevant information from the Web of Data, such as pluviometric data, agricultural data, market prices etc. might become available through the radio platform.

The radio platform described in this paper not only facilitates production, consumption and management of voice-and web-based radio content, but it also enables access to the Web for people who do not have a computer or the Internet.

Contributions of this paper are:

- A radio platform with both a web and a voice-based mobile interface that allows content creation, retrieval and indexing of spoken radio content.

\footnotetext{
${ }^{5}$ UNMD, United Nations Millenium Declaration, General Assembly resolution 55/2. United Nations, New York, 2000

${ }^{6}$ Communication possibilities with people living in the diaspora, are described by Serigne Mansor Tall in: Les émigrés sénégalais et les nouvelles technologies de l'information et de la communication. http://www.unrisd.org
} 
- African community radio, introduced as a new interface to the Web of Data

This paper is structured as follows. In section 2 we describe related work. In section 3 the architecture of the radio platform is described, the use cases collected from three different radios in Mali, as well as the principles used to manage the content. In section 4 we describe the challenges related to the organization of the voice-based radio content. In section 5 we discuss future work that must be done on the Web of Radios, including the sustainability aspects.

\section{Related Work}

Related work on the development of a similar platform was done in the Freedom Fone ${ }^{7}$. Freedom Fone is a project initiated by The Kubatana Trust of Zimbabwe, a civil and information activist platform from Zimbabwe. Freedom Fone is open source software for creating audio content using phone. Freedom Fone provides a voice platform similar to the basic setup proposed in this paper, but without the Linked Data enabled data management.

Research on speech recognition started in the 1930s and resulted in commercial deployments of voice-based services in the 1970s. Major achievements on language recognition, mainly for English, took place in the 1980s and 1990s and culminated in the development of VoiceXML by the W3C Voice Browser group, in 1999, facilitating and standardizing the development of voice applications [3].

Sheetal Agarwal et al. from IBM Research India, developed a system to enable authorship of voice content for $2 \mathrm{G}$ phone in a web space, they named the WWTW or World-Wide Telecom Web. The system is not connected to the Web, therefore not allowing access by third party search engines. The system represents closed web space, within the phone network. Especially the lack of open search possibility constrains its growth [4].

From Burundi a system has been reported [5] to use tagging software and multimedia mobile data collection. The software is named EthnoCorder ${ }^{8}$. The NGO that co-developed this app was Help Channel Burundi. However, because of the current unavailability of multimedia devices in the given rural context, this technical solution may be still out of reach of community radio stations targeted in this study.

A related project on the Semantic XO and Linked Data for developing countries is described by Guéret et al. [6]. The Semantic XO is a system that connects rugged, low-power, low-cost robust small laptops (aka the XO promoted by the One Laptop Per Child organization) for the empowerment of poor communities, based on Linked Data principles in order to publish previously unpublished data.

De Boer et al. [2] describe a distributed voice- and web-based market information system, named Radio Marché, aimed at stimulating agricultural trade in rural areas of Africa. This system connects to regionally distributed market information systems, using Linked Data approaches.

\footnotetext{
${ }^{7}$ Freedom Fone, http://www.freedomfone.org

${ }^{8}$ http://www.ethnocorder.com/
} 


\section{The radio platform}

The design of the radio platform described in this section is based on extensive use case and requirements analysis, performed in Mali, with the collaboration of radio journalists from community radio stations. The research was done as part of the Foroba Blon $^{9}$ project $^{10}$, funded by the International Press Institute, and the VOICES project ${ }^{11}$, partially funded by the EU, within the 7th Framework Programme. The Foroba Blon project is aimed at supporting and promoting citizen journalism in developing countries. The VOICES project is aimed at developing innovative mobile voice services to support users in underprivileged communities in African countries.

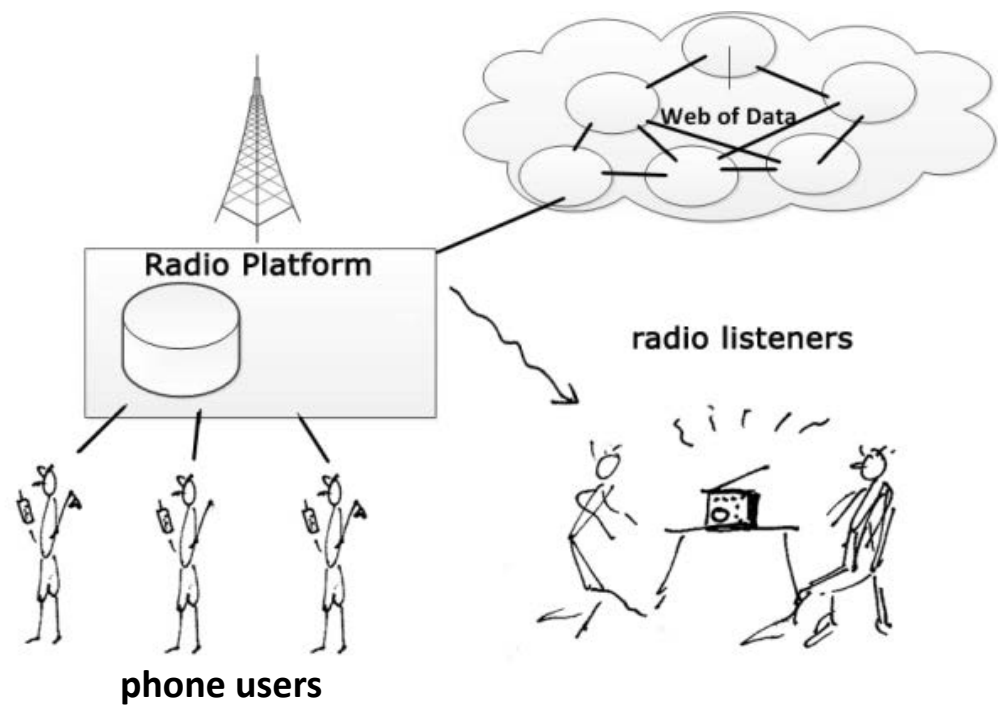

Fig. 1. Conceptual design of the radio platform as a voice-interface to the Web for people who are out of reach of computers and the Internet, but do have phone or radio.

\footnotetext{
${ }^{9}$ Foroba Blon in Bambara language refers to a large space, where everyone has the right to speak in front of the village chief; the truth must be told here, but only respectfully, without insulting anyone.

${ }^{10}$ Foroba Blon, Citizen Journalism: http://worldplantage.wordpress.com/2012/01/14/communityradio-in-tominian-and-segou-mali/

${ }^{11}$ VOICES: http://www.mvoices.eu
} 


\subsection{Community radio in Mali and how it operates}

In Mali many community radio stations exist. Some are state funded and connected to the national broadcasting service ORTM (Office Radio Télévision du Mali). Others are privately funded or completely self-supportive. According to their business, funding scheme, size and location some radio stations do have computers and internet, some have computers without internet connection and some do not have any computer facilities at all. All these radio stations are situated within the coverage area of mobile telephony.

The Malian community radios have large bases of listeners and the radius of coverage ranges between $100-200 \mathrm{~km}$. These radio stations create their own programs and broadcast local and regional news, music, informative programs, round table programs and paid announcements. Three radio station stations are involved in the projects described in this paper. These are: Radio ORTM Ségou, a state owned radio, that has computers and a 2 Mbps fixed line (DSL) internet connection. Radio ORTM Ségou broadcasts programs in French and Bambara, the most widely spoken language in Mali.

The second radio station is Radio Moutian, in Tominian. This radio is independent and its funding is based on paid airtime for announcements and private gifts from third parties. Radio Moutian has a computer but no internet connectivity. Programs are mainly broadcasted in Bomu, a local language fro the Tominian region. The third radio is Radio Seno in Bankass. This radio is independent from the Malian state and has only analogue equipment. There are no computers, there is no internet connection here, but the radio has many listeners in the region around Bankass. The main language spoken here is Dogon. The activities of the three above mentioned radio stations are related to three types of end-users or customers:

- NGOs, that buy airtime to broadcast public announcements about informative and educational topics, such as agriculture and public health information. This type of service is usually based on fixed monthly subscriptions to airtime for recurring broadcasts.

- Non-commercial listeners from the region, who buy a few minutes of airtime and pay a broadcast fee per minute airtime. The information is usually brought to the radio, or communicated via phone and subsequently written down on paper by the radio staff. Some listeners call in on a given time slot (one hour per week) and leave a short voice message ( few seconds only) as a reaction to a program that was broadcast on a certain popular topic. These messages are named letters to the editors (LTE).

- Journalists or trusted village reporters that phone to the radio and leave local news or interviews on a regular base. In the current situation, all incoming phone calls are attended by a radio staff member and annotated in tabular form on paper.

\subsection{The radio platform architecture}

The proposed radio platform, which we named Foroba Blon (FB), consists of a data store containing recorded voice messages and related meta-information. 
The interface to the FB radio platform is either purely voice-based, through mobile phone, for entering new content, or web-based. Users of the mobile interface are the listeners from the region, who enter letters to the editor (LTE). These people only have mobile phones but no access to the Internet. Their calls are answered by the system with a pre-recorded welcome message in a local Malian voice inviting them to leave their message. For the sake of user-friendliness, the user interface and the dialogue for this category of users is kept as short and simple as possible, since the expected callers will be unfamiliar with interactive voice response systems and may not respond to a complex computer-generated dialogue asking to press buttons etc.

Another category of users of FB are the trusted reporters calling from the field, and also using the mobile interface. They phone in and leave their spoken report for broadcasting. These users are previously registered, having their phone number, name, address and preferred language in FB. These users will be trained to navigate the voicemenu, and use the IVR system, asking to press a button on the phone to confirm or answer a question about their current location, subject of the message, etc. The FB system always answers the registered caller in his/her preferential language.

The voice messages are stored as audio files in the FB data store, together with metainformation being the date and time of the call, the length of phone call in seconds, the phone number of the caller. Messages from trusted users are linked to the owner, his/her address, and his/her preferred language. For all users of the system, confidentiality and anonymity will be ensured, according to the broadcast policies used by the radio stations in Mali.

The FB radio platform also has a normal web interface, where internet-connected end-users/customers can access and upload a voice message. Depending of their customer relationship to the radio, they can login to the radio-platform as (i) registered users such as NGOs, and trusted reporters, or (ii) as unregistered users. There is an option to sign up and create a user account by registering the name, phone number, village and preferred language. Unregistered users can access former broadcasts since this is public information.

For the radio user, FB provides a web-based interface, enabling them to manage the data in the data store. It provides a file list where they can access, listen, broadcast, delete files, and add/update/delete meta-information.

The radio station that has no computer nor internet, has only a very limited interface to the radio platform, since this is the constraint of a voice interface. The radio user receives a welcome message asking if she wants to hear the last 10 messages, or if she wants to manage the welcome messages to the end-users.

The FB radio platform is hosted either locally, on a stand-alone computer, or in the cloud. The FB consists of a voice platform running an open source web server and a local voice browser that handles the voice interaction. The FB radio platform uses a GSM gateway device, e.g. OfficeRoute, ${ }^{12}$ a device that handles incoming and outbound calls and streams the voice messages to and from the phone.

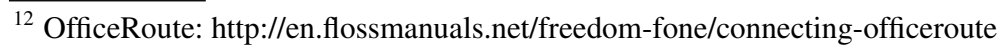


The FB radio platform could in theory be physically hosted anywhere in the world, on any web server, connected to the Internet. However, in this actual case in rural regions of Mali, this is not possible. Firstly, the radio platform has to be accessible using an inexpensive local Malian phone number, so it must be connected to a Malian phone network. Secondly, the web service accessed over the Internet must also be accessible locally. Since the internet connectivity in Mali is usually of low bandwidth and of high latency, voice web services hosted in datacenters in the US or Europe, are too slow for proper deployment in Mali. For these two reasons, the system has to be preferably hosted locally in Mali. In the absence of good and reliable datacenters or hosting providers in Mali, the radios can decide to deploy the FB radio platform on a local computer at their own premises. Obviously before this can be done, the radio staff members have to be trained how to do operational maintenance of the FB platform, and especially how they can cope with frequent power outages, and bring the system back to a consistent state

\section{Organizing the radio content}

The next challenge is how to manage the spoken content of un-resourced languages such as Bomu, Bambara and Dogon. Since up to present no interactive voice response (IVR) systems exist for these languages, the voice-based content cannot be indexed by conventional search engines. Therefore collecting as much meta-information as possible is essential. Very simple ways of indexing the messages are based on owner (known through phone number) automatic language recognition, time slot, (e.g. all messages collected on January 13 between 10 and 11 a.m. are related to the radio program on harvesting sheanuts). The radio journalist can manually enter meta-information such as keywords, village region, language, name or any other attribute to an audio file using her radio-web interface. In the future existing tagging systems such as EthnoCorder may be considered, to facilitate meta-data collection.

\subsection{Prepare the data for unexpected re-use}

For the moment, the data of the radio platform will be only used locally, but we want to prepare the platform for the future. According to Tim Berners-Lee it is the unexpected re-use of information which is the great value, added by the Web. We will use Linked Data principles for the audio data on the FB radio platform, as following the golden rules by Berners-Lee [7] for publishing and connecting data on the Web, while adhering to its architecture and standards: 1 . Using URIs as names for things; 2. Using HTTP URIs so that people can look up those names; 3. Providing useful information, using the standards like RDF, SPARQL; 4. Including links to other URIs, so that people can discover more things, especially across regions and future similar community radio platforms. If the FB radio platform proves to be a success, other instances of FB may be installed at local radio stations in Mali, across borders, in neighbouring countries, Burkina Faso, Ghana, Senegal, Guinée where conditions with regards to illiteracy, local languages, mobile telephony and community radio are similar to those in Mali. This will create a Web of African community radios that are linked to each other and that will eventually become part of the Web of Data. 


\subsection{Organize an open source community of developers to create applications for the radio platform}

In the VOICES and Foroba Blon projects one instance of the radio platform is developed by a small team of developers, in collaboration with end-users ${ }^{13}$ sponsored by the International Press Institute as a pilot project. However, to enable further development of the radio platform, and to expand the scale of the web of radios, it is important to look at new ways of production and consumption of data and services. African community radios operate in a low-income region where the sustainability of a system relies on the underlying business model. Community radios do not have enough earnings to invest in new systems, and their listeners-base is large, but poor. Application development will therefore to be organized in a cost-effective way. We propose to organize an open source community of developers and to rely on commons-based peer production for the development of applications that will open the Web of Data to radio using voice-modality.

\section{Discussion and future work}

From this paper it becomes clear that the Web of African Radios can only emerge as an interface to the Web of Data, when sufficient applications are built, that link voicebased content. For the navigation of voice menus and other voice-based dialogues small subsets of the local languages such as Bambara and Bomu have to be recorded and resourced using time-consuming techniques and efforts. The user interfaces have to be extensively tested and validated with end-users in the local situation, since these are culturally sensitive. For the resourcing of more local languages crowd-sourcing techniques may be applied. The issue of meta-information is another important topic. In the model presented for the FB radio platform in this paper, only a small amount of meta-data is collected. When the repositories of spoken content start to become larger, new innovative ways of describing spoken content have to be developed. To contribute to a critical mass of content and applications that are necessary in this rural domain, a socio-technical network has to be put in place, that must be supported by a community of contributors: web developers, listeners that provide meta-information, local ICT-entrepreneurs, people who are willing to produce and consume data. According to Kazman and Hong-Mei Chen [8] organizing a community of developers around an open source service requires a consolidated kernel infrastructure, allowing peripheral services to be created by a de-centralized community of developers. Specific social and technical mechanisms are needed to ensure long-term participation and to encourage community engagement. In this case this is justified by the aim to open the Web of Data to people who are out of reach of computers and the internet.

\section{References}

1. Akkermans, H., Grewal, A., Bon, A., Tuyp, W., Allen, M., Gyan, N.B.: W4RA-VOICES

${ }^{13}$ At the moment of writing, the use cases have been collected in Mali, and the FB platform is being built accordingly. However, no feed-back has yet been received from the users 
eld report. Tech. rep., Web Alliance for Regreening Africa (2011), http: //www.mvoices.eu/2011/03/25_Voices-W4RA_Public_Report.pdf

2. De Boer, V., De Leenheer, P., Bon, A. Gyan, N.B., Van Aart, C., Guéret, C., Tuyp, W., Boyera, S., Allen, M., Akkermans, H. Radio Marché: Distributed Voice en Web Interfaced Market Information System under Rural Conditions, in prep.

3. W3C: Voice Extensible Markup Language VoiceXML Version 2.0, W3C Recommendation 16 March (2004), http://www.w3.org/TR/voicexml20/

4. Agarwal, S.K., Jain, A., Kumar, A., Rajput, N.: The world wide telecom web browser. In: Proceedings of the First ACM Symposium on Computing for Development. ACM DEV 10, (2010) New York, NY, USA, ACM 4:14:9.

5. Horst, N.: EthnoCorder in Burundi: innovation, data collection and data use. In: Participatory learning and action. IIED (2011). http://pubs.iied.org/pdfs/14606IIED.pdf

6. Guéret, C., Schlobach, S.: SemanticXO : connecting the XO with the worlds largest information network. In: Proceedings of the First International Conference on eTechnologies and Networks for Development, ICeND2011. (2011)Communications in Computer and Information Science, Springer LNCS.

7. Berners-Lee T. : Linked Data, the four rules (2006). http://www.w3.org/DesignIssues/LinkedData.html

8. Kazman, R. \& Hong-Mei, C.: The Metropolis Model. A new logic for development of crowdsourced systems. Communications of the ACM, (2009), Vol. 52, No 7. 